\title{
Fast jedes politische Vorhaben ist heute auch ein IT-Projekt
}

\author{
Rede des Bundesministers der Innern, Dr. Wolfgang Schäuble, zur Eröffnung der Konferenz* \\ „Advancing E-Government“ am 1. März 2007 in Berlin
}

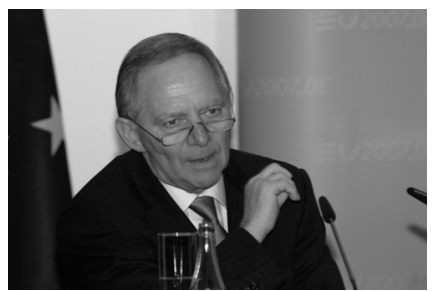

Dr. Wolfgang Schäuble, Bundesminister des Innern.
Ich begrüße die Teilnehmer aus 30 europäischen Nationen. Sie alle sind Expertinnen und Experten für E-Government aus Verwaltung, Wirtschaft und Zivilgesellschaft.

Die europäische Einigung ist eine Erfolgsgeschichte für uns alle. Wir Europäer haben heute durch Integration, Bündelung gemeinsamer Interessen und gemeinsames Handeln einen so sicheren Frieden und so großen Wohlstand erreicht wie nie zuvor in der Geschichte. Ich bin davon überzeugt, dass wir - wenn wir in der Welt des 21. Jahrhunderts bestehen wollen - nicht weniger, sondern mehr Europa brauchen.

Die Geschichte der europäischen Einigung begann mit der Schaffung eines gemeinsamen Wirtschaftsraumes in Europa. Für mich als Innenminister ist Europa heute aber vor allem auch ein Raum der Freiheit, der Sicherheit und des Rechts. Diesen Raum gilt es auszubauen.

Während der deutschen EU-Ratspräsidentschaft wollen wir die hierzu schon vorhandenen Mittel verbessern. Die paneuropäische Zusammenarbeit der Staaten soll intensiviert werden. Für mich ist insbesondere von Bedeutung, dass es uns gelingt, gemeinsam mit der Europäischen Kommission und den Mitgliedstaaten neue Impulse für die künftige Gestaltung der europäischen Innenpolitik zu geben.

Mittlerweile ist ein weiterer Raum entstanden: Europa als elektronischer Kommunikationsraum im Internet. Die Kommunikation und Zusammenarbeit im Internet prägen die Entwicklung der europäischen Integration und der grenzüberschreitenden Zusammenarbeit in großem Maße. Charakteristisch für den elektronischen Kommunikationsraum Europa sind meines Erachtens drei Aspekte.

Erstens hat der Kommunikationsraum Internet völlig neue Formen von Kommunikation und Zusammenarbeit ermöglicht. Im Web werden heute rund um die Uhr europaweit Informationen abgerufen, Geschäfte getätigt und Nachrichten ausgetauscht.

Oder nehmen Sie die Auktionsplattform ebay: In Europa hat ebay 50 Millionen Mitglieder, die im 2. Quartal 2006 insgesamt 596 Millionen Artikel eingestellt haben - statistisch gesehen hat jeder Europäer eine Auktion angemeldet. Mehr als 170.000 Menschen in Europa verdienten im letzten Jahr ihren Lebensunterhalt ganz oder teilweise mit dem Handel bei ebay.

Unter dem Schlagwort „Web 2.0“ haben Internet-Technologien in den letzten Jahren völlig neue Formen sozialer Interaktion möglich gemacht. Hilfsmittel wie Wikis, Blogs oder Podcasts eröffnen den Internet-Nutzern Gestaltungsmöglichkeiten jenseits traditioneller Kanäle der Nachrichtenverteilung und des Austauschs.
Nach den Bombenschlägen von London im Juli 2005 gab es beispielsweise schon innerhalb der ersten zwei Stunden mehr als 1.300 Schilderungen von Augenzeugen in so genannten Blogs im Internet - weitaus detaillierter und umfangreicher als die Berichterstattung der Nachrichtenagenturen.

Die Nutzerinnen und Nutzer agieren heute über entsprechende Plattformen im Internet selbst unmittelbar und schnell. Ein gutes Beispiel ist auch die Online-Enzyklopädie Wikipedia, die in Deutschland mit über 500.000 Einträgen schon mit klassischen Enzyklopädien konkurrieren kann.

Die Technologie erlaubt neue Formen der Interaktion, die wiederum neue Tätigkeitsfelder und Geschäftsmodelle ermöglichen und in zahlreichen Wirtschaftszweigen substanzielle Veränderungsprozesse bewirkt haben oder noch bewirken werden.

Mehrere Millionen Menschen engagieren sich beispielsweise schon heute in der Online-Community „Second Life“. Da wundert es nicht, dass große Markenartikel-Hersteller dort virtuelle Geschäfte eröffnen. Mit Schweden wird der erste Staat dort in Kürze eine - virtuelle - Botschaft eröffnen.

Das bringt mich zu dem zweiten Aspekt, den ich hier skizzieren möchte: der Bedeutung von Informationstechnik und InternetTechnologien für die Aufgabenerfüllung des Staates und die Umsetzung politischer Vorhaben.

Fast jedes politische Projekt enthält oder verursacht heute auch ein IT-Projekt. Ich habe das in Deutschland in den letzten Jahren intensiv verfolgt. Ob Sie eine Arbeitsmarktreform auf den Weg bringen oder Straßenbenutzungsgebühren für Lkw einführen oder das Gesundheitswesen modernisieren wollen: Für die Umsetzung der Projekte sind IT-Vorhaben unentbehrlich und damit auch erfolgskritisch.

Das gilt im Übrigen auch für politische Vorhaben der Europäischen Union. Ich will nur zwei Beispiele aus der europäischen Sicherheitspolitik nennen, die ich für wichtig halte:

Die Aufhebung der Kontrollen an den Binnengrenzen Europas ist ohne ein funktionierendes Schengener Informationssystem nicht denkbar. Das so genannte SIS hält die Polizeibehörden über mit Haftbefehl gesuchte oder an der Einreise zu hindernde Personen sowie über gestohlene Fahrzeuge europaweit auf dem Laufenden. Hier gehört der europaweite Zugriff auf einen gemeinsamen, ständig aktuell gehaltenen Datenbestand bereits zum Arbeitsalltag der Behörden der Vertragsstaaten. SIS wird derzeit weiterentwickelt. Die Bundesregierung begrüßt die Erweiterung des Informations- und Fahndungssystems um wichtige Sicherheitselemente wie Lichtbilder und Fingerabdrücke.

Ein anderes Beispiel ist das VISA-Informationssystem. Damit werden zukünftig die Daten über erteilte, abgelehnte, widerrufene oder annullierte Visa einschließlich der biometrischen Daten der betroffenen Personen europaweit bei Visumerteilung

Die Konferenz wurde von der Bundesregierung im Rahmen der deutschen EU-Ratspräsidentschaft veranstaltet. 
und Einreisekontrolle verfügbar sein. Das so genannte VIS wird wesentlich zur besseren Bekämpfung illegaler Migration und des Visa-Shopping, aber auch des internationalen Terrorismus und der Organisierten Kriminalität beitragen.

Aufgabe von E-Government ist es, die sich ständig fortentwickelnden Möglichkeiten der Informations- und Kommunikationstechnologie in unsere staatliche Aufgabenerfüllung einzubringen. Nicht nur die europaweit tätigen Unternehmen entwickeln für sich und ihre Kunden einen europäischen Informations- und Kommunikationsraum. Auch die öffentlichen Stellen aller Mitgliedstaaten müssen an diesem Informations- und Kommunikationsraum mitwirken und ihn für ihre Zwecke nutzen. Wie gut ein Staat Informations- und Kommunikationstechnologie für seine Arbeit einsetzt, bestimmt mit über die Qualität, mit der seine Bürgerinnen und Bürger in Europa leben, arbeiten und wirtschaften.

Die EU-Dienstleistungsrichtlinie (zu der uns Herr Stoll gleich etwas mehr berichten wird) ist für die deutsche Verwaltung und deren Informations- und Kommunikationstechnologie eine große Herausforderung. Nach dem europäischen Binnenmarkt für Waren, von dem wir alle seit Jahren profitieren, geht es jetzt um die Errichtung eines europäischen Marktes für Dienstleistungen und somit darum, die Erbringung von Dienstleistungen in anderen Mitgliedstaaten der Europäischen Union so einfach wie möglich zu machen.

Die Nutzung von IT und Internet wird hier von der Europäischen Union sehr konsequent einbezogen: Dienstleistungserbringer können nach der Richtlinie in den Mitgliedstaaten so genannte „One-Stop-Shops“ erwarten, über die sie alle Formalitäten elektronisch abwickeln können.

Die Umsetzung der Dienstleistungsrichtlinie läuft für ein föderal organisiertes Gemeinwesen wie Deutschland wahrscheinlich auf ein beträchtliches Umbauprogramm auf allen Ebenen hinaus. Wir müssen alle verwaltungsinternen Prozesse und Schnittstellen umfassend überprüfen, um einheitliche Ansprechpartner und elektronische Prozesse zu gewährleisten. Gleichwohl halte ich die Richtlinie für eine große Chance, einen elektronischen Kommunikationsraum Europa auch für die Verwaltungen der Mitgliedstaaten zu realisieren.

Ein dritter Aspekt ist heute in diesem elektronischen Kommunikationsraum wesentlich: die Internet-Sicherheit. Ganz gleich ob bei elektronischen Verfahren zur Umsetzung der Dienstleistungsrichtlinie, ob beim E-Business oder beim Surfen: Immer müssen unsere Bürger vor Manipulation, Verlust und unberechtigter Kenntnisnahme ihrer elektronischen Daten und ihrer Kommunikationen geschützt werden.

Als Innenminister betrachte ich die Sicherheitslage im Internet mit großer Sorge. Sowohl im Bericht des Bundesamtes für Sicherheit in der Informationstechnik zur Lage der IT-Sicherheit in Deutschland als auch im aktuellen Symantec-Report ist nachzulesen, dass die Zahl der Schwachstellen und IT-Angriffe weiterhin stetig zunimmt.

Allein in der ersten Jahreshälfte 2006 wurden etwa 2250 neue IT-Schwachstellen dokumentiert. Das entspricht einem erneuten Anstieg von 18 Prozent gegenüber dem zweiten Halbjahr 2005. Der Anteil von Spam-Nachrichten beträgt inzwischen 60 bis 90 Prozent am gesamten E-Mail-Verkehr. Dazu kommen immer öfter Phishing-Attacken und Bot-Netze. Im ersten Halbjahr 2006 stieg die Zahl der Phishing-Geschädigten in Deutschland laut
BITKOM nochmals um bis zu 50 Prozent. Der aktuelle volkswirtschaftliche Schaden durch Phishing dürfte nach Schätzungen der BITKOM mittlerweile im zweistelligen Millionenbereich liegen.

Der erste bei ebay verkaufte Artikel war ein kaputter Laserpointer. Zwar erschließt sich mir der Sinn dieses deals nicht, aber ich gehe davon aus, dass es sich um ein legales Geschäft handelte. Das kann man von vielen Geschäften, die heute über das Internet abgewickelt werden, leider nicht sagen.

Der größte Teil der Betrügereien im Internet besteht aus Täuschungen über die Identität. Wenn man im Internet kommuniziert oder handelt, kann man sich nicht unmittelbar von der Identität und Vertrauenswürdigkeit des Gegenübers überzeugen. Phishing oder ebay-Betrügereien setzen auf dieses Manko der virtuellen Realität: Betrüger handeln unter der Internet-Kennung oder den Passwörtern anderer Menschen, um Geschäfte zu deren Lasten zu tätigen.

Die Sicherheit der privat wie beruflich genutzten Computer und Netzwerke entwickelt sich immer mehr zu der Schlüsselfrage unserer Informationsgesellschaft. Wir können IT-Sicherheit nur gemeinsam gewährleisten. Die gemeinsame Verantwortung von Wirtschaft und Staat für die Sicherheit in der Informations- und Kommunikationstechnologie und des Internets wird Schwerpunkt der europäischen IT-Sicherheitskonferenz im Juni 2007 sein, zu der ich während der deutschen Präsidentschaft einlade.

Die Sicherheit im Internet ist zugleich ein Thema für die EGovernment-Politik: Wie sich die öffentliche Verwaltung, wie sich Regierungen und Behörden im Internet verhalten, wie sie staatliche Leistungen und Informationsangebote bereitstellen, haben sie gestaltenden und prägenden Charakter für die Sicherheit im Internet. Dessen müssen wir uns alle bewusst sein.

Die Mitgliedstaaten der Europäischen Union müssen sich gemeinsam um den europäischen Kommunikationsraum kümmern. Das Internet ist notwendig international. Wie im Internet kommuniziert und gehandelt wird, ist national zunehmend weniger zu beeinflussen. Mit Blick auf den elektronischen Kommunikationsraum Europa sehe ich vier große Ziele für das europäische E-Government:

Erstens müssen die Mitgliedstaaten der Europäischen Union die Dienstleistungen ihrer öffentlichen Verwaltungen an den privaten und beruflichen Bedürfnissen der Nutzerinnen und Nutzer ausrichten. Andernfalls werden unsere Angebote die Zielgruppe nicht erreichen. Das haben wir in Deutschland in den letzten Jahren teilweise schmerzlich erfahren. Erfolgreiche E-BusinessAngebote zeichnen sich durch hohe Nutzerorientierung aus. Genauso ist es im E-Government.

Wenn die Verwaltung - wie es häufig noch der Fall ist - zu wissen glaubt, was die Welt brauche, ist sie im Zeitalter von „Web 2.0“ auf dem Holzweg und verschleudert Ressourcen. Die Einbeziehung der Nutzerinnen und Nutzer sowie die Gestaltung kooperativer Online-Angebote müssen E-Government-Standard werden. Das bedeutet insbesondere, dass wir diese Technologien in unseren Behördenalltag integrieren müssen. Das können WikiLösungen für unser Informations- und Wissensmanagement sein oder die Nutzung von modernen Suchmaschinen statt komplizierter Verwaltungshandbücher.

Zweitens müssen nutzerorientierte E-Government-Angebote die Bürokratielast verringern. In der EU-Initiative „i2010“ haben 
wir uns verpflichtet, die Bürokratielast in Europa um 25 Prozent zu senken.

Deutschland hat mit der Einführung des Standardkostenmodells in der Bundesverwaltung einen wichtigen Schritt zur Erfüllung dieses Ziels gemacht. Alle Behörden der Bundesverwaltung haben die aus der Gesetzgebung entstehenden Informationspflichten erfasst. Wir sind dabei, die daraus folgenden Belastungen in Euro und Cent zu messen. Mit dem Standardkostenmodell wurden in den Niederlanden Belastungen durch staatliche Informationspflichten in Höhe von rund 16 Mrd. Euro festgestellt. Für Deutschland ist ebenfalls ein erhebliches Potenzial für den Abbau bürokratischer Bürden zu erwarten.

Auf der heutigen Konferenz wollen wir verschiedene Strategien und Lösungen der Mitgliedstaaten und der Europäischen Kommission zum Abbau von Bürokratie durch E-Government vorstellen und sie mit den Entwicklungen in nicht-europäischen Staaten vergleichen.

Drittens ist jeder Erfolg in Sachen Bürokratieabbau auch ein Fortschritt im so genannten Lissabon-Prozess, mit dem wir die Europäische Union zu einem der attraktivsten Orte zum Leben, Arbeiten und Investieren auf der Welt machen wollen. Der Lissabon-Prozess kann durch leistungsfähige, serviceorientierte und wirtschaftliche Verwaltungen gefördert werden. Mit professioneller IT in den Behörden können wir es unseren Unternehmen leichter machen und damit den Wirtschaftsstandort Europa voranbringen.

Dazu sollte die Weiterentwicklung der IT in den öffentlichen Verwaltungen der Mitgliedstaaten koordiniert erfolgen. Durchgängig elektronische Zusammenarbeit lässt sich nur mit europaweit akzeptierten Standards realisieren. Gerade diese gehören zu den ,dicken Brettern“, die gebohrt werden müssen.

Am 24. November 2005 unterschrieben die Teilnehmer der Ministerkonferenz „Transforming Public Services“ die so genannte Manchester-Deklaration. In den letzten 15 Monaten sind wir meines Erachtens noch nicht weit gekommen. Das Thema Standardisierung ist weiterhin eine der großen paneuropäischen E-Government-Baustellen. Wir dürfen die Arbeiten an der elektronischen Verwaltungszusammenarbeit im europäischen Binnenmarkt nicht auf die lange Bank schieben. Der globale Wettbewerb lässt uns keine Zeit.

Die deutsche EU-Ratspräsidentschaft setzt sich für eine Harmonisierung der Standards ein. Ein besonders wichtiges Thema sind standardisierte, offene Dokumentenaustauschformate in Europa. Wenn wir zwischen den Behörden oder zwischen Unternehmen und Behörden elektronisch ohne Medienbrüche zusammenarbeiten wollen, müssen dafür Datenformate zur Verfügung stehen, die für alle Beteiligten ohne besondere Kosten, ohne Rechte Dritter und über Jahre bis Jahrzehnte verwendbar sind.
Davon sind wir heute noch ein weites Stück entfernt. Die faktische Dominanz herstellerabhängiger Dokumentenformate und die unklare Situation bei der internationalen Standardisierung zeigen es.

Viertens halte ich eine europäische Standardisierung gerade bei der elektronischen Identifizierung für erforderlich. Bürgerinnen und Bürger sowie Unternehmen sollten in Europa bis 2010 eine sichere elektronische Identifizierungsmöglichkeit bei ihren Kontakten mit Behörden im eigenen Land oder in einem anderen Mitgliedstaat nutzen können. Jeder Mitgliedstaat sollte bis 2010 eine interoperable elektronische Identifizierung und Beglaubigung elektronischer Dokumente bereitstellen. Damit würden wir nicht nur das grenzüberschreitende E-Government befördern, sondern auch den Identitätsmissbrauch im Internet zurückdrängen.

Gestern haben zu beiden Themen - Dokumentenaustauschformate und Identifizierungstechnologien - Fach-Workshops stattgefunden. Die Ergebnisse der Workshops werden in die heutige Konferenz eingebracht. Heute ist zu besprechen, welche Anstrengungen der Mitgliedstaaten und der Kommission hierzu notwendig sind und welche Schritte auf dem Weg zu interoperablen Behördendienstleistungen nun konkret gemacht werden müssen.

Beide Themen sind ein Beispiel dafür, wie die europäischen Staaten den Kommunikationsraum Internet gestalten. Das Handeln der Staaten kann Vorreiter für verständliche und sichere Kommunikation im Internet sein. Das fördert E-Business und E-Government gleichermaßen.

Diese Erkenntnis hat Deutschland in seiner E-GovernmentStrategie aufgegriffen. Erst gestern hat die Bundesregierung den Umsetzungsplan zum Programm „E-Government 2.0“ beschlossen. Wichtige Schwerpunkte sind E-Identity-Konzepte sowie integrierte elektronische Prozessketten zwischen Unternehmen und Behörden.

Mir ist wohl bewusst, dass europaweite Standardisierungsprozesse und solide, effiziente Kooperationen im E-Government einen langen Atem brauchen. Wir brauchen Kontinuität, um nachhaltige Resultate zu erreichen.

Im Rahmen unserer Trio-Präsidentschaft mit Portugal und Slowenien wollen wir für mehr Nachhaltigkeit in den Projekten sorgen, die auf Konferenzen wie der heutigen von den Mitgliedstaaten der Europäischen Union auf den Weg gebracht werden. Aus diesem Grund begrüße ich besonders herzlich die Teilnehmerinnen und Teilnehmer aus Portugal und Slowenien, die auch mit eigenen Beiträgen in einem Panel und im Plenum vertreten sein werden.

Ich bin mir sicher, dass wir in unserer Trio-Präsidentschaft das Ziel erreichen, die heute entwickelten Lösungsansätze auch über die deutsche Ratspräsidentschaft hinaus umsetzen zu können. 